\title{
Editorial: Supporting institutional change
}

\author{
Cathy Malone \\ Middlesex University London
}

In a sector not known for its flexibility or ability to respond rapidly to technological change (Killen 2015) the papers in this section demonstrate a range of dynamic and successful institutional responses to the challenges of the pandemic. They reveal the processes and thinking by which so many institutions responded rapidly to the pandemic and brought about changes to practice at scale. Setting the lens at the level of institution or large faculty provides a particular perspective on the change management process and these reflections provide an insight into how large numbers of staff and students were supported to engage in a new online learning and teaching environment.

The challenges the pandemic presented here include the need to facilitate the mass shift to online learning, the need to rapidly digitally enable large staff groups while maintaining the quality of provision, and the institutional commitment to principles of accessibility and inclusivity.

Many of the papers in this section note the significant increase in engagement with all avenues and means of support and CPD, where participation, website views and resource downloads appeared to expand exponentially; small communities of practice were thrust into the spotlight and bespoke courses for small groups now needed to be delivered institution wide. The international range of authors and case studies included here tells us something about the global ubiquity of the challenge. There are also commonalities in both principle and process in how institutions responded to the task of managing institutional change and upskilling large staff groups.

A common theme is the commitment to pedagogy and educational theory; when supporting institutional shift in mode of delivery, pedagogy has been prioritised over technology. Many authors note that while the online pivot was sudden and unplanned, the response needed to be more measured and intentional: there was a need to avoid the 
temptation to mechanically translate face to face teaching approaches to online activities and processes. Rather, it was important to revisit core educational principles such as UDL (Universal Design for Learning) to more deliberately select how to teach online or in a hybrid mode. In practical terms this meant turning staff into conscious learning designers and to support them move between modalities (online, face to face and increasingly a mixture of the two) in an intentional way, able to distinguish between learning objectives and mode dependent instructional objectives.

At the same time the human needs of staff at the heart of this change project are repeatedly noted. Frequent reference is made to the diversity of the staff groups involved and the need to acknowledge and address the anxieties and lack of confidence manifested by many. This very diversity has implications for the differentiated practical response. Some patterns in practice begin to emerge; workshops and online resources, blog posts and videos provide a consistent message concerning guiding principles and expectations, while tips, checklists and templates provide a range of ideas for staff to select from - these modes were followed up with opportunities for closer peer support and hands on practice. Combining different modes of CPD delivery enabled small teams to deliver support at scale that includes choice and feels targeted and bespoke. Working at a similar scale but focusing on mass delivery of student learning development during the pandemic, we see two case studies which outline the development of online resource repositories and how increased usage brought about significant benefits to local learner development teams.

Summing up, the papers in this section provide an exciting insight into teams who collaborated under pressure and for whom the pandemic proved a positive catalyst for change. The results of their work captured here demonstrate a creative response to the pandemic and as well as offering exciting blueprints for practice and development, are inspiring in the openness they demonstrate to new ways of learning and supporting institutional change.

\section{References:}

Killen, C. (2015) 'Enhancing the student digital experience: A strategic approach', JISC report available online, https://www.jisc.ac.uk/full-guide/enhancing-the-digital-studentexperience (Accessed 22 October 2021). 